\title{
Lack of Appetite Visual Analogue Scale
}

National Cancer Institute

\section{Source}

National Cancer Institute. Lack of Appetite Visual Analogue Scale. NCI Thesaurus. Code C141571.

A lack of appetite scale from 0 to 10 on which the patient marks the current level of lack of appetite experienced. 